\title{
Bioactive Constituents of Methanol Extract of Xylopia aethiopica (UDA) Fruits from Nsukka, Enugu State, Nigeria
}

\author{
Innocent Uzochukwu Okagu*, Uchenna Ekpereamaka Ngwu, Chinyere Jane Odenigbo \\ Medical Biochemistry, Toxicology and Drug Discovery Unit, Department of Biochemistry, University of Nigeria, Nsukka, Nigeria \\ Email: ^innocent.okagu@unn.edu.ng
}

How to cite this paper: Okagu, I.U., Ngwu, U.E. and Odenigbo, C.J. (2018) Bioactive Constituents of Methanol Extract of Xylopia aethiopica (UDA) Fruits from Nsukka, Enugu State, Nigeria. Open Access Library Journal, 5: e4230.

https://doi.org/10.4236/oalib.1104230

Received: December 3, 2017

Accepted: March 2, 2018

Published: March 5, 2018

Copyright $\odot 2018$ by authors and Open Access Library Inc.

This work is licensed under the Creative Commons Attribution International License (CC BY 4.0).

http://creativecommons.org/licenses/by/4.0/

\begin{abstract}
The bioactive compounds in methanol extract of Xylopia aethiopica fruits were determined using a combination of gas chromatography and mass spectrometry (GC-MS). The compound identification was based on the molecular structure, molecular mass and calculated fragments. Interpretation on mass spectrum of GC-MS analyzer (Hewlett Packard GC-MS system) was done using the database of National Institute Standard and Technology (NIST). The results showed different peaks representing the presence of about 58 bioactive compounds which are mainly carboxylic acids, esters, phenolic compounds and fatty acids. The most abundant bioactive compounds detected were 2,4,6-octatriene (2.74\%), cyclohexanemethanol (2.57\%), kaurene (3.59\%), 9,12-octadecadienoic acid (5.63\%), 1,6-cyclodecadiene (10.81\%), terpineol (3.22\%), cyclohexene (3.32\%), copaene (3.04\%), 1,6-cyclodecadiene (5.53\%), 1-hexadecyne $(5.63 \%)$ and silane $(4.63 \%)$. The presence of these bioactive components suggests that the extract is of great pharmaceutical value.
\end{abstract}

\section{Subject Areas}

Biological Chemistry, Medicinal Chemistry

\section{Keywords}

Xylopia aethiopica, Bioactive Compounds, Gas Chromatography, Mass Spectrometry, Methanol Extract

\section{Introduction}

Africa is blessed with lots of medicinal plants that contain active principles that make them useful to cure Man's diseases. Medicinal plants are locally accepted 
because they are believed to have less or no side effects when compared to synthetic drugs. They are also available and cheap to obtain [1]. The analysis of the chemical constituents of these medicinal plants would be necessary in determining and isolating the active principles of these plants and hence, making way for the assessment of their biological activities. Currently, there are lots of techniques that can be employed to identify these constituents of plants such as chromatographic and spectroscopic techniques. A combination of gas chromatography (GC) and mass spectrometry (MS) has over the years proven to be one of the best combinational techniques in the identification of the chemical constituents of various plants. While gas chromatography separates the constituents, mass spectrometry helps to determine the molecular weight of these compounds [2]. The GC also separates volatile components in a sample while MS fragments the components and identifies them on the basis of their mass. Separation in GC is based on their boiling point where the substances with higher boiling point come out later and those with lower boiling point come out first. When they are out, they go into the MS which identifies them using their mass to charge ratio [3]. Xylopia aethiopica is a plant that is widely spread in the tropical Africa, especially in Ghana, Zambia, Mozambique and Angola. It is abundant in lowland rainforest and most fringe forest in the savanna zones of Nigeria [4]. It matures as a slim, tall tree of approximately $60 \mathrm{~cm}$ in diameter and up to $30 \mathrm{~m}$ high with a straight stem having a slightly stripped or smooth bark [5]. The taxonomical classification of Xylopia aethiopica includes:

\begin{tabular}{|c|c|c|}
\hline Kingdom & $\rightarrow$ & Plantae \\
\hline Subkingdom & $\rightarrow$ & Viridiplantae \\
\hline Infra-kingdom & $\rightarrow$ & Streptophyta \\
\hline Super-division & $\rightarrow$ & Embryophyta \\
\hline Division & $\rightarrow$ & Tracheophyta \\
\hline Subdivision & $\rightarrow$ & Spermatophytina \\
\hline Class & $\rightarrow$ & Magnoliopsida \\
\hline Superorder & $\rightarrow$ & Magnolianae \\
\hline Order & $\rightarrow$ & Magnoliales \\
\hline Family & $\rightarrow$ & Annonaceae \\
\hline Genus & $\rightarrow$ & Xylopia L \\
\hline Species & $\rightarrow$ & Xylopia aethiopica \\
\hline
\end{tabular}

Xylopia aethiopica has been recorded to have so many medicinal and nutritional values such as treatment of sores, boils, cough, wounds and cuts, among others [5]. [6] reported that fruits of the plant are used by herbalists for increasing menstrual flow and for terminating unwanted pregnancy. It is also added as a stimulant to several other herbal preparations in traditional medicine for treating stomach ache, bronchitis, dysentery, neuralgia and biliousness. Several biological activities such as promotion of prostaglandin synthesis [7], hypertensive and diuretic effects as well as antimicrobial, antimalarial and anti-parasitic activities have been reported of the plant [8]. Some of the chemical constituents of this plant include resins, annonacin, reberoside, avicien, rebersole, alkaloids, 
tannin, oxalate and flavonoids [9]. Anonaceine, an alkaloid similar to morphine was found in the essential oil from $X$. aethiopica [10]. The antifertility effect of the plant has also been reported [8]. The plant has been shown to elevate intraocular pressure [11], induce hepatic [12] and renal cell damages [13], and decreases sperm quality [8]. The major bioactive components of $X$. aethiopica fruits varied based on the location of source [14]: that from Cameroun were $\beta$-phellandrene $+1,8$-cineole (31\%), $\beta$-pinene (8\%) and $\alpha$-pinene (3.4\%) [15] while that from Ivory Coast was $\beta$-pinene (20.56\%) [16]. In Chad, the major constituents were $\alpha$-pinene (5.56\%), $\beta$-pinene (24.6\%), $\beta$-phellandrene $(12.36 \%)$ and $\alpha$-phellandrene (7.16\%) [17]. From Togo, $\alpha$-pinene (23.6\%), $\beta$-pinene (11\%), sabinene $(9.8 \%)$, germacrene $\mathrm{D}(8.3 \%)$ and 1,8 -cineole $(8.2 \%)$ were detected by [18]. In Sudan, the GC-MS results revealed the presence of monoterpenes (78.58\%): monoterpene hydrocarbons $(42.31 \%)$ and oxygenated monoterpenes (36.27\%) and sesquiterpenes (18.85\%): sesquiterpene hydrocarbons ((15.88\%) and oxygenated sesquiterpenes (2.97\%) [19]. It is also believed that solvent extraction affects the chemical content of plant materials; hence, this study was therefore designed to determine the bioactive compounds of methanol extract of Xylopia aethiopica fruits from Enugu State, Nigeria using gas chromatography-mass spectrometry (GC-MS) technique.

\section{Materials and Methods}

\subsection{Collection, Authentication and Extraction of Plant Material}

The plant material used in this study, dried fruits of Xylopia aethiopica (Figure 1), was collected from Ogige Main-Market in Nsukka, Enugu State, Nigeria in the month of March, 2017. It was authenticated at the Herbarium of the Department of Plant Science and Biotechnology, University of Nigeria, Nsukka, Enugu State,

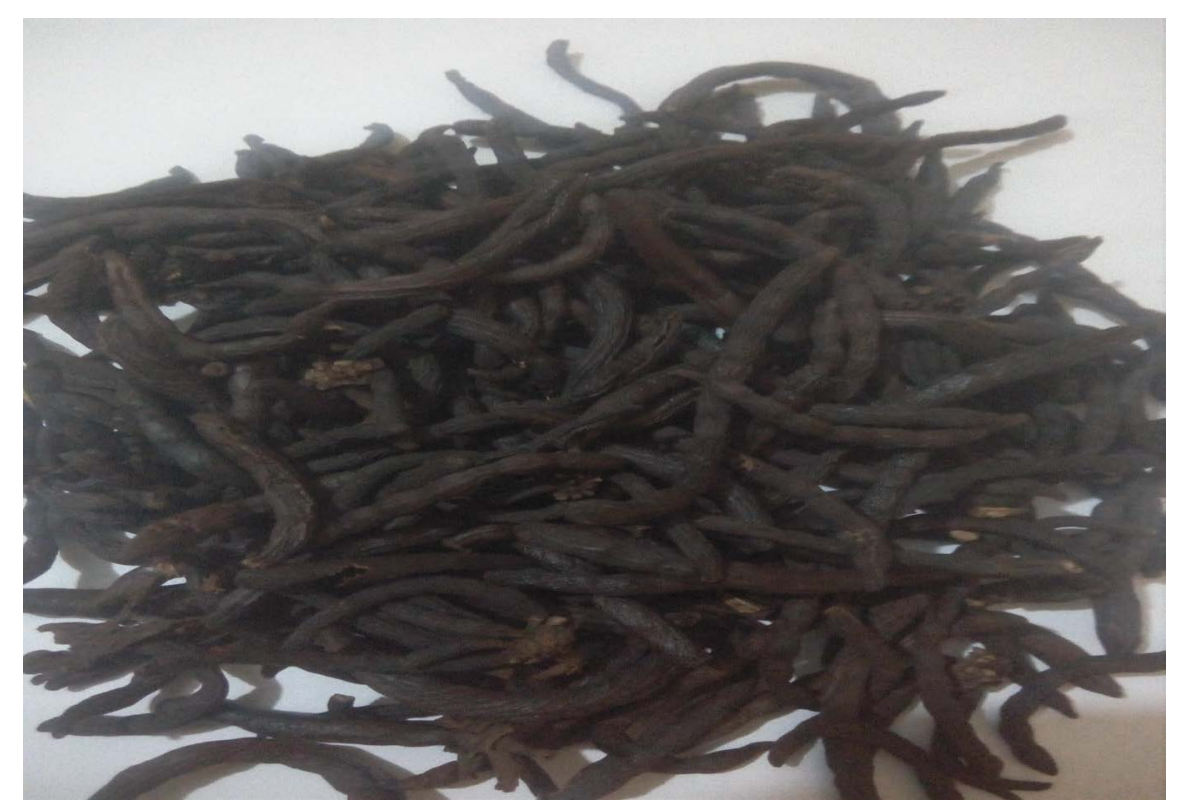

Figure 1. Xylopia aethiopica fruits. 
Nigeria. The dried fruits were ground into powder using a mechanical grinder. The powdered sample (1000 g) was soaked in 4.5 litres of methanol (JHD, China) in an air-tight container and was left for 48 hours with occasional stirring. The suspension was filtered and the filtrate concentrated using a rotary evaporator under reduced pressure at $40^{\circ} \mathrm{C}$. The oily dark brown concentrate of percentage yield of $17.8 \%$ was subjected to GC-MS analysis.

\subsection{Determination and Identification of Bioactive Compounds in Methanol Extract of Xylopia aethiopica Fruits}

The bioactive components of methanol extract of Xylopia aethiopica fruits were determined using a GC-MS analyzer (Hewlett Packard GC-MS system with Purge and Trap, US EPA 8260/5035 by GC/MSD and ASTM D2600/D2908). The fused silica HP-20 M polyethylene glycol column $(50 \mathrm{~m} \times 0.2 \mathrm{~mm}, 0.2 \mu \mathrm{m}$ thickness) was directly coupled to the mass spectrometer. The carrier gas was helium $(1 \mathrm{~mL} / \mathrm{min})$. The program used was $4 \mathrm{~min}$ isothermal. The injection port temperature was $250^{\circ} \mathrm{C}$ and the detector temperature $280^{\circ} \mathrm{C}$. Mass spectra were taken at $70 \mathrm{eV}$; a scan interval of $0.5 \mathrm{~s}$ and fragments from 45 to $450 \mathrm{Da}$. The relative percentage amount of each component was calculated by comparing its average peak area to the total area. Software adopted to handle mass spectra and chromatograms was Turbomass version 5.2.0. Identification of the compounds were based on the molecular structure, molecular mass and calculated fragments. Interpretation of mass spectrum from GC-MS was conducted using the database of National Institute Standard and Technology (NIST) having more than 82,000 patterns. The name of each bioactive component of the extract was ascertained and the percentage amount of each was calculated by comparing its average peak area to the total area. The spectrum of unknown components was compared with the spectrum of the known components stored in the NIST library. This was done in order to determine whether this extract contains any individual compound or group of compounds, which may substantiate its ethno-medicinal applications.

\section{Results}

\section{Bioactive Compounds in Methanol Extract of Xylopia aethiopica Fruits}

The identities of the bioactive compounds in methanol extract of Xylopia aethiopica fruits were confirmed based on the peak area and retention time and presented in Table 1. The GC-MS analysis of methanol extract of $X$. aethiopica fruits revealed fifty eight compounds representing $99 \%$ of the total extract and ten compounds representing only $1 \%$ unidentified. The major constituents were 2,4,6-octatriene (2.74\%), cyclohexanemethanol (2.57\%), kaurene (3.59\%), 9,12-octadecadienoic acid (5.63\%), 1,6-cyclodecadiene (10.81\%), terpineol (3.22\%), cyclohexene (3.32\%), copaene (3.04\%), 1,6-cyclodecadiene (5.53\%), 1-hexadecyne (5.63\%) and silane (4.63\%). 
Table 1. Bioactive compounds in methanol extract of Xylopia aethiopica fruits.

\begin{tabular}{|c|c|c|c|}
\hline $\begin{array}{l}\text { Peak } \\
\text { No. }\end{array}$ & $\begin{array}{l}\text { Retention } \\
\text { Time }\end{array}$ & $\begin{array}{c}\text { Area } \\
\%\end{array}$ & Identified compound \\
\hline 1. & 4.361 & 3.22 & Terpineol, cis- $\beta$-terpineol cyclohexene, 1-methyl-3-(1-methylethenyl) \\
\hline 2. & 4.533 & 1.07 & 1,6-Octadien-3-ol, 3,7-dimethyl-bicyclo [2.2.1] hept-2-ene, 2,7,7-trimethyl-1,6-Octadien-3-ol, 3,7-dimethyl \\
\hline 3. & 4.751 & 2.74 & 2,4,6-Octatriene, 2,6-dimethyl-, (E, Z), 2,4,6-Octatriene, 3,4-dimethyl- \\
\hline 4. & 4.905 & 0.43 & $\begin{array}{l}\text { Tricycle }[4.4 .0 .0(2,8)] \text { dec-4-ene 1,3-cyclohexadiene, 5-(3-butene-1- } \gamma \text { 1), Bicyclo [3.1.0] hex-2-ene, } \\
\text { 4-methylene-1-(1-methylethyl)- }\end{array}$ \\
\hline 5. & 5.019 & 0.34 & Bicyclo [6.1.0] non-1-ene cyclohexanone, 5-methyl-2-(1-methylethlidene)-2-methyl-1-octen-3-yne \\
\hline 6. & 5.088 & 0.37 & 2-(5-Aminohexyl) furan ethanone, 1-(methylenecyclopropyl) 3-Methylenecyclohexene \\
\hline 7. & 5.174 & 1.69 & 3-cyclohexen-1-ol , 4-methyl-1-(1-methylethyl)- \\
\hline 8. & 5.266 & 1.59 & $\begin{array}{l}\text { Cyclohexane, 1-methylene-3-(1-methylethyl)-, (R)-, } 3 \text { cyclohexene-1-methanol, } \alpha, \alpha \text { 4-trimethyl-, cyclohexene, } \\
\text { 5-methyl-3-(1-methylethenyl) -, trans-(-)- }\end{array}$ \\
\hline 9. & 5.317 & 0.44 & Bicyclo [3.1.1] hept-2-ene-2-methanol, 6,6-dimethyl-, cis-bicyclo [3.3.0] oct-2-ene, BIcyclo (3.2.1) oct-2-ene \\
\hline 10. & 6.032 & 0.57 & Benzenemethanol, 4-(1-methylehyl) \\
\hline 11. & 6.484. & 3.32 & Cyclohexene, 4-ethynyl-4-methyl-3-(1-methylethenyl) -1-(1-methyethyl)-(3R-trans)-, (+)-4-Carene \\
\hline 12. & 6.582 & 1.37 & $\alpha$-cubebene \\
\hline 13. & 6.862 & 3.04 & Copaene, $\alpha$-Cubebenecopaene \\
\hline 14. & 6.982 & 2.84 & $\begin{array}{l}\text { 1H-cyclopropa [a] naphthalene, 1a,2,3,5,6,7,7a,7b-Octahydro-1,1,7,7a-tetramethyl-, [1aR-(1a } \alpha, 7 \alpha, 7 \mathrm{a} \alpha, 7 \mathrm{~b} \alpha)] \text {-, } \\
\text { Bicyclo [4.4.0] dec-1-ene, 2-isopropyl-5-methyl-9-methylene- }\end{array}$ \\
\hline 15. & 7.137 & 0.49 & $\begin{array}{l}\text { 3H-3a, 7-Methanoazulene, 2,4,5,6,7,8-hexahydro-1,4,9,9-tetramethyl-,[3aR-(3a } \alpha, 4 \beta, 7 \alpha) \text {, 3H-3a, 7-Methanoazulene, } \\
\text { 2,4,5,6,7,8-hexahydro-1,4,9,9-tetramethyl-,[3aR- }(3 \mathrm{a} \alpha, 4 \beta, 7 \alpha)] \delta \text {-selinene }\end{array}$ \\
\hline 16. & 7.303 & 5.53 & $\begin{array}{l}\text { 1,6-cyclodecadiene, 1-methyl-5-methylene-8-(1-methylethyl)-, [s-(E,E)]-1H-cyclopenta [1,3] cyclopropa[1,2] } \\
\text { benzene, octahydro-7-methyl-3-methylene-4-(1-methyethyl)-, [3aS-(3a } \alpha, 3 \mathrm{~b} \beta, 4 \beta, 7 \alpha, 7 \mathrm{aS} \text { )]-, } \\
\text { Bicyclo [4.4.0] dec-1-ene, 2-isopropyl-5-methyl-9-methylene- }\end{array}$ \\
\hline 17. & 7.406 & 3.76 & $\begin{array}{l}\text { (+)-Epi-bicyclosesquiphellandrene } 1 \mathrm{H} \text {-cyclopenta }[1,3] \text { cyclopropa }[1,2] \text { benzene, } \\
\text { octahydro-7-methyl-3-methylene-4-(1-methylethyl)-, [3aS-(3a } \alpha, 3 \mathrm{~b} \beta, 4 \beta 7 \alpha, 7 \mathrm{aS} *)]- \text {, Naphthalene, } \\
\text { 1,2,3,4,4a,5,6,8a-octahydro-7-methyl-4-methylene-1-(1-methylethyl)-, } 1 \alpha, 4 \mathrm{a} \beta, 8 \mathrm{a} \alpha)\end{array}$ \\
\hline 18. & 7.537 & 2.34 & $\begin{array}{l}\text { Naphthalene, 1,2,3,4,4a,5,6,8a-octahydro-7-methyl-4-methylene-1-(1-methylethyl)-, } 1 \alpha, 4 \mathrm{a} \beta, 8 \mathrm{a} \alpha) \text {-, Bicyclo[4.4.0] } \\
\text { dec-1-ene, 2-isopropyl-5-methyl-9-methylene- }\end{array}$ \\
\hline 19. & 7.640 & 2.65 & $\begin{array}{l}\text { Naphthalene, 1,2,3,4,4a,5,6,8a-octahydro-7-methyl-4-methylene-1-(1-methylethyl)-, 1 } \alpha, 4 \mathrm{a} \beta, 8 \mathrm{a} \alpha) \text {-, Naphthalene, } \\
\text { 1,2,4a,5,6,8a-octahydro-7-methyl-4-methylene-1-(1-methylethyl)-, }\end{array}$ \\
\hline 20. & 7.709 & 0.79 & $\begin{array}{l}\text { 1,6-cyclodecadiene, 1-methyl-5-methlylene-8-(1-methylethyl)-, [s-(E,E)]-, 1H-cyclopenta }[1,3] \text { cyclopropa [1,2] ben- } \\
\left.\left.\text { zene, octahydro-7-methyl-3-methylene-4-(1-methylethyl)-, [3aS-(3a } \alpha, 3 \mathrm{~b} \beta, 4 \beta, 7 \alpha, 7 \mathrm{aS} \mathrm{S}^{*}\right)\right]-\end{array}$ \\
\hline 21. & 7.921 & 10.81 & $\begin{array}{l}\text { 1,6-cyclodecadiene, 1-methyl-5-methlylene-8-(1-methylethyl)-, [s-(E,E)]-, } 1 \mathrm{H} \text {-cyclopenta }[1,3] \text { cyclopropa }[1,2] \\
\left.\left.\text { benzene, octahydro-7-methyl-3-methylene-4-(1-methylethyl)-, [3aS-(3a } \alpha, 3 \mathrm{~b} \beta, 4 \beta, 7 \alpha, 7 \mathrm{aS} \mathrm{S}^{*}\right)\right]- \\
\text { (+)-Epi-bicyclosesquiphellandrene }\end{array}$ \\
\hline 22. & 8.046 & 1.10 & $\begin{array}{l}\text { Bicyclo [4.4.0] dec-1-ene, 2-isopropyl-5-methyl-9-methylene-, (+)-Epi-bicyclosesquiphellandrene, Naphthalene, } \\
\text { 1,2,3,4,4a,5,6,8a-octahydro-7-methyl-4-methylene-1-(1-methylethyl)-, } 1 \alpha, 4 \mathrm{a} \alpha, 8 \mathrm{a} \alpha)\end{array}$ \\
\hline 23. & 8.109 & 1.78 & $\begin{array}{l}\text { Naphthalene, 1,2,4a,5,6,8a-hexahydro-4,7-dimethyl -1-(1-methylethyl)-, 1,6-cyclodecadiene, } \\
\text { 1-methyl-5-methylene-8-(1-methylethyl)-, [s-(E,E)]-, Naphthalene, } \\
\text { 1,2,3,4,4a,5,6,8a-octahydro-7-methyl-4-methylene-1-(1-methylethyl)-, 1 } \alpha, 4 \mathrm{a} \alpha, 8 \mathrm{a} \alpha)\end{array}$ \\
\hline 24 & 8.212 & 1.76 & $\begin{array}{l}\text { Cyclohexene, 6-ethenyl-6-methyl-1-(1-methylethyl)-3-(1-methyethylidene)-, (S)-, Copaene, Naphthalene, } \\
1,2,3,4,4 \mathrm{a}, 5,6,8 \mathrm{a} \text {-octahydro-7-methyl-4-methylene-1-(1-methylethyl)-, } 1 \alpha, 4 \mathrm{a} \alpha, 8 \mathrm{a} \alpha)\end{array}$ \\
\hline
\end{tabular}




\section{Continued}

\begin{tabular}{|c|c|c|c|}
\hline 25. & 8.247 & 2.47 & Naphthalene, 1,2,3,5,6,8a-hexahydro-4,7-dimethyl -1-(1-methylethyl)-(1S-cis) \\
\hline 26. & 8.355 & 0.71 & 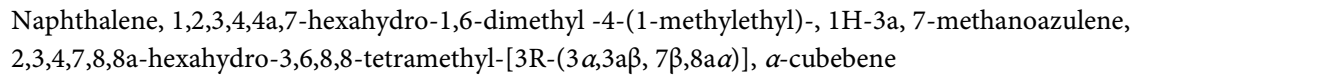 \\
\hline 27. & 8.395 & 0.34 & 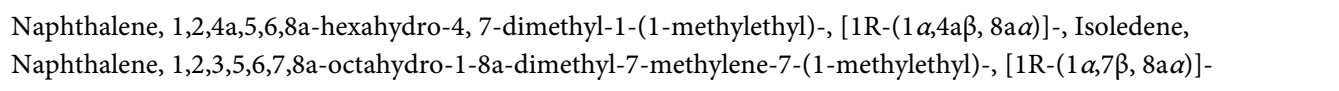 \\
\hline 28. & 8.521 & 2.57 & Cyclohexanemethanol, 4-ethenyl- $\alpha, \alpha, 4$-trimethyl-3-(1-methylethenyl)-[1R-( $1 \alpha, 3 \mathrm{a}, 4 \beta)]$ \\
\hline 29. & 8.573 & 0.59 & $\begin{array}{l}\text { 1H-Indene, 2,3-dihydro-2,2-dimethyl 4, 4-dimethyl-3-(3-methybut-3-enylidine)-2-methylenbicyclo [4.1.0] } \\
\text { heptane, 1H-Indene, 2,3-dihydro-2,2-dimethyl 4, 4-dimethyl- }\end{array}$ \\
\hline 30. & 8.807 & 0.43 & $\begin{array}{l}\text { 1-Hydroxy-1, 7-dimethyl -4-isopropyl -2, 7-cyclodecadiene, Bicyclo [2.2.1] heptan-2-ol, 1,3,3-trimethyl-, acetate, } \\
\text { (1S-exo)-, ethanone, 1-(2-methyl-2-cyclopenten-1-y1)- }\end{array}$ \\
\hline 31. & 8.859 & 0.90 & $\begin{array}{l}\text { Tricyclo [5.2.2.0 (1,6)] undecan-3-ol, 2-methylene-6,8,8-trimethly-, Tricyclo [4.4.0.0(2,7)] dec -3-ene-3-methanol, } \\
\text { 1-methyl-8-(1-methylethyl)-, 1H-cycloprop [e] azulen-7-ol, decahydro-1,1,7-trimethyl-4-methylene-, } \\
{[1 \mathrm{ar}-(1 \mathrm{a} \alpha, 4 \mathrm{a} \alpha, 7 \beta, 7 \mathrm{a} \beta, 7 \mathrm{~b} \alpha)]}\end{array}$ \\
\hline 32. & 8.928 & 0.46 & 2-(4a,8-demethyl-1,2,3,4,4a,5,6,7-octahydro-naphthalen-2-yl)-prop-2-en-1-ol, Patchoulene $\gamma$ Gurjunenepoxide-(2) \\
\hline 33. & 9.156 & 1.14 & $\begin{array}{l}\text { 4,4-Dimethyl-3-(3-methylbut-3-enylidene)-2-methylenbicyclo [4.1.0] heptanes, ledene oxide-(II), } \\
\text { 8-Quinolinol, 4-methyl- }\end{array}$ \\
\hline 34. & 9.254 & 0.49 & $\begin{array}{l}\text { Cyclohexene, 6-ethenyl-6-methyl-1-(1-methyethyl)-3-(1-methylethylidene)-, (S)-, 4,7-Methanoazulene, } \\
\text { 1,2,3,4,5,6,7,8-Octahydro-1,4,9,9-tetramethyl-[1S-(1 } \alpha, 4 \alpha, 7 \alpha)], \text { Naphthalene, } \\
\text { 1,2,3,5,6,8a-hexahydro-4,7-dimethyl-1-(1-methylethyl)-, (1S-cis)- }\end{array}$ \\
\hline 35. & 9.351 & 3.25 & $\begin{array}{l}\text { 1H-cycloprop[e]azulen-7-ol, decahydro-1,1,7-trimethyl-4-methylene-, [1ar-(1a } \alpha, 4 \mathrm{a} \alpha, 7 \beta, 7 \mathrm{a} \beta, 7 \mathrm{~b} \alpha)]-(-) \text {-spathulenol, } \\
\text { 1,7,7-Trimethyl-2-vinylbicyclo [2.2.1]hept-2-ene }\end{array}$ \\
\hline 36. & 9.431 & 0.48 & Aromadendrene, cedren-13-ol, 8-thujopsene-13 \\
\hline 37. & 9.488 & 0.77 & $\begin{array}{l}\text { Aromadendrene oxide-(2), 2-(4a, 8-dimethyl-1,2,3,4,4a,5,6,7-octahydro-naphthalen-2-yl)-prop-2-en-1-ol, } \\
\text { longipinocarveol, trans- }\end{array}$ \\
\hline 38. & 9.614 & 1.68 & $\begin{array}{l}\text { 1H-Indole-3-carboxylic acid, 4-hydroxy-,6-isopropenyl-4, 8a-dimethyl-1,2,3,5,6,7,8,8a-octahydro-naphthalen-2-ol, } \\
\text { Naphthalene, 1,2,3,4-tetrahydro-1, 6-dimethyl-4-(1-methylthyl)-, (1S-cis)- }\end{array}$ \\
\hline 39. & 9.877 & 0.60 & $\begin{array}{l}\text { Cycloisolongifolene, 8-hydroxy-, endo-, isolongifolene, 7,8-dehydro-8a-hydroxy-, 2-oxa-1,3-disilacyclohexane, } \\
\text { 1,1,3,3,-tetramethyl- }\end{array}$ \\
\hline 40. & 9.958 & 3.06 & $\begin{array}{l}\text { Cycloisolongifolene, 8,9-dehydro-Cycloisolongifolene, 8-hydroxy-, endo-, 10-oxatricyclo [4.2.1.1(3,9)] dec-4-ene, } \\
\text { 9-ethenyl- }\end{array}$ \\
\hline 41. & 10.221 & 0.37 & $\begin{array}{l}\text { 7R, 8R-8-Hydroxy-4-isopropylidene-7-methylbicyclo [5.3.1] undec-1-ene, isolongifolene, } \\
\text { 9,10-dehydro-Neoisolongifolene, 8,9-dehydro- }\end{array}$ \\
\hline 42. & 10.427 & 0.45 & Benzoic acid, 2-amino-3-hydoxy-phenol, 4-methyl-2-nitro-thiourea, N-ethyl-N'-tricyclo[3.3.1.1 (3,7)]dec-1-yl- \\
\hline 43. & 10.581 & 0.46 & $\begin{array}{l}\text { Tricycle [4.4.0.0(2,7)] dec-3-ene-3-methanol, 1-methyl-8-(1-methylethyl)-, } \\
\text { calarene epoxide 1-(3,3-Dimethyl-1-1-yl)-2,2-dimethylcyclopropene-3-carboxylic acid }\end{array}$ \\
\hline 44. & 10.719 & 0.37 & $\begin{array}{l}\text { Cyclooctene, 4-methylene-6-(1-propenenylidene)-Isolongifolene, } \\
\text { 7,8-dehydro-8a-hydroxy-4-(2-Nitrophenylimino)) -2-pentanon }\end{array}$ \\
\hline 45. & 10.764 & 0.51 & $\begin{array}{l}\text { 1H-cycloprop[e]azulen-7-oL, decahydro-1,1,7-trimethyl-4-methylene-, [1 } 1 \mathrm{ar}-(1 \mathrm{a} \alpha, 4 \mathrm{a} \alpha, 7 \beta, 7 \mathrm{~b} \alpha)] \text {, acetic acid, } \\
\text { 6,6-dimethyl-2-methylene-7-(3-oxobutylidene) oxepan-3-yl methyl ester, isolongifolene, 7,8-dehydro-8a-hydroxy- }\end{array}$ \\
\hline 46. & 10.999 & 0.58 & Aromadendrene oxide-(1), Alloaromadendrene oxide-(2) Glaucyl alcohol \\
\hline 47. & 11.119 & 0.34 & $\begin{array}{l}\text { 2-Butenal, 2-methyl-4-(2,6,6-trimethyl-1-cyclohexen-1-yl)-, } \\
\text { 2-(4a,8-dimethyl-1,2,3,4,4a,5,6,7-octahydro-naphthalen-2-yl)-prop-2-en-1-ol, isoaromadendrene epoxide }\end{array}$ \\
\hline
\end{tabular}




\section{Continued}

\begin{tabular}{|c|c|c|c|}
\hline 48. & 11.296 & 0.42 & Benzeneethanol, 2-methoxy- $\alpha$-methyl-(+)-2-carene, 4- $\alpha$-isopropenyl-, Boldenone \\
\hline 49. & 11.428 & 0.41 & $\begin{array}{l}\text { (-)-Neoclovene-(I), dihydro-1H-Cycloprop[e]azulene, decahydro-1,1,4,7-tetramethyl-, [1aR-(1a } \alpha, 4 \beta, \\
4 \mathrm{a} \beta, 7 \beta, 7 \mathrm{a} \beta, 7 \mathrm{~b} \alpha)], 1 \text {-cyclohexene, 1,3,3-trimethyl-2-(1-methylbut-1-en-3-on-1-yl)- }\end{array}$ \\
\hline 50. & 11.617 & 0.59 & 3-Heptadecene, (Z)-, 8-Heptadecene, n-Nonadecanol-1 \\
\hline 51. & 11.657 & 0.61 & $\begin{array}{l}\delta \text {-Gurjunenepoxide-(2), 9-isoprop-yl-1-methyl-2-methylene-5-oxatricyclo [5.4.0.0 }(3,8)] \text { undecanecyclohexane, } \\
\text { 1,5-diethenyl-3-methyl-2-methylene-, }(1 \alpha, 3 \alpha, 5 \alpha\end{array}$ \\
\hline 52. & 12.510 & 2.62 & n-Hexadecanoic acid \\
\hline 53. & 13.179 & 0.63 & 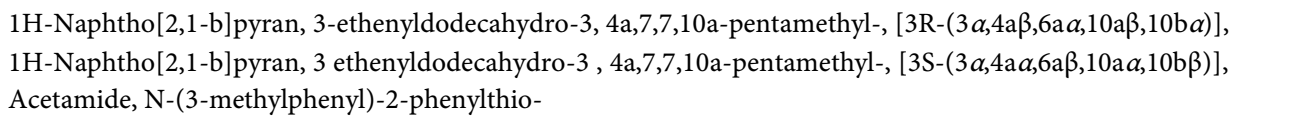 \\
\hline 54. & 13.499 & 3.59 & Kaur-16-ene, Kaurene \\
\hline 55. & 14.198 & 5.63 & 9,12-Octadecadienoic acid (z,z)-, 1-Hexadecyne \\
\hline 56. & 14.358 & 0.58 & Octadecanoic acid \\
\hline 57. & 15.508 & 4.78 & $\begin{array}{l}\text { Silane, dimethyl (3-methylbut-2-enyloxy) heptyloxy-, } \\
\text { Galaxolide } 1 \text { (3E, 5E, 7E)-6-Methyl-8-(2.6.6-trimethyl-1-cyclohexenyl) -3,5,7-octatrien-2-one }\end{array}$ \\
\hline 58. & 16.263 & 3.08 & $\begin{array}{l}\text { Naphthalene, decahydo-1,1, 4a-trimethyl-6-methylene-5-(3-methylene-4-pentenyl)-, [4aS-(4a } \alpha, 5 \alpha, 8 \mathrm{a} \beta)] \\
\text { Benz [c] acridine, 7,9-demethyl-3-Methyl-1-phenyl-2-azafluorene. }\end{array}$ \\
\hline
\end{tabular}

\section{Discussion}

This study demonstrated the usefulness of GC-MS as a valuable tool for the identification of bioactive compounds present in plant materials. The analysis of the methanol extract of $X$. aethiopica fruits revealed the presence of 58 bioactive compounds. Among the identified compounds, the presence of fatty acids like n-hexadecanoic acid, 9,12-octadecanoic acid, hexadecanoic acid and methyl ester may be associated with the reported antioxidant property of the plant [16] [20]. Also, the presence of these fatty acids and esters in the extract suggests that it can serve as a pesticide, an anti-androgenic flavor, and $\alpha$-reductase inhibitor [21]. It can also serve as cancer preventive, [22], hepatoprotective, antieczemic, hypocholesterolemic and anticoronary agent [23]. In traditional practice, the fruits are crushed and mixed with Shea butter and used as body creams or perfumes [5]. This is could be due to the presence of carboxylic acids such as octadecanoic acid and esters such as acetic acid, 6,6-dimethyl-2-methylene-7-(3-oxobutylidene) oxepan-3-yl methyl ester in the plant [23]. Xylopia aethiopica has been applied in traditional medicines as an effective antimicrobial agent [5]. This antimicrobial activity could be due to the presence of some aliphatic alcoholic compounds like octadiene-3-ol, which has earlier been shown to possess antimicrobial effects [24]. The compound, 1,6-cyclodecadiene, otherwise known as Germacrene D, was also identified by [25]. The compound is a class of volatile organic hydrocarbons, which plays a role as a precursor of various sesquiterpenes such as cadinenes and selinenes [26]. Reports showed that Germacrene D has insecticidal activity against mosquitoes [27], antibacterial activity [28], as well as repellent activity against aphids [29] and ticks [30]. [25] also detected the presence of spathulenol, an oxygenated sesquiterpene that has immunomodulatory 
effects, mosquito repellant activity, antimicrobial and anti-inflammatory activities [31]. This could be the reason why essential oil from the plant is used as mosquito and housefly repellents, and termite antifeedant in traditional practice [32]. Xylopia aethiopica has been reported to be of help in enhancing the healing of some ailments in man [33] and this may be associated with the presence of phenolic compounds such as 2-amino-3-hydroxy phenol as detected in extract in the present study and by [24]. Xylopia aethiopica has been used as flavour pepper and spice in food and for management of liver diseases [34]. This could be as a result of the compound, terpineol, cis- $\beta$-terpineol cyclohexene, 1-methyl3-(1-methylethenyl) which is a citrus essential oil. Citrus essential oils could be considered suitable alternatives to chemical additives for use in various industrial areas such as food, cosmetics and pharmaceuticals. They also have an attractive and mild aroma that can mask food smells from fish, meat, garlic and onion. These seem to be positive functions for use as industrial antimicrobial agents such as preservatives. Cyclohexanones and cyclohexanols such as bicyclo [6.1.0]non-1-ene-cyclohexanone, 5-methyl-2-(1-methylethlidene)-2-methyl-1-octen-3yne, 3-cyclohexen-1-ol, 4-methyl-1-(1-methylethyl)-, and others identified in the plant can be used as solvents for paints and dyes, in pesticides, and as an intermediate for pharmaceuticals, films, soaps, and coatings [35]. Naphthalene identified in the plant is said to be hazardous [36]. This could be the reason excessive consumption of the plant has been reported to be toxic [13]. This study has also shown the presence and amount of bioactive components in plant materials depend on the location of the plant source.

\section{Conclusion}

The present study identified 58 bioactive compounds in the methanol extract of Xylopia aethiopica fruits using gas chromatography-mass spectrometry (GC-MS). This plant has many bioactive compounds that possess antioxidant, anti-inflammatory, anti-microbial and anticancer properties. This showed that the plant extract should be of great pharmaceutical interest. Haven identified many bioactive compounds in methanol extract of Xylopia aethiopica fruits in the present study, and it is recommended that the active ingredients are isolated and subjected to further tests to compare their usefulness in the prevention and treatment of various conditions.

\section{Conflict of Interest}

The authors declare no conflict of interest.

\section{References}

[1] Ashokkumar, R. and Ramaswamy, M. (2014) Phytochemical Screening by FTIR Spectroscopic Analysis of Leaf Extracts of Selected Indian Medicinal Plants. International Journal of Current Microbiology and Applied Sciences, 3, 395-406.

[2] Murugan, M. and Mohan, V.R. (2014) Phytochemical, FT-IR and Antibacterial Activity of Whole Plant Extract of Aerva lanata (L.) Juss. Ex. Schult. Journal of Medi- 
cinal Plants Studies, 2, 51-57.

[3] Ashish, C., Manish, K.G. and Priyanka, C. (2014) GC-MS Technique and Its Analytical Applications in Science and Technology. Journal of Analytical and Bioanalytical Techniques, 5, 6-10.

[4] Eze, K.N. (2012) Antifertility Effects of Ethanolic Extract of Xylopia aethiopica on Male Reproductive Organ of Wistar Rats. American Journal of Medicine and Medical Sciences, 2, 12-15.

[5] Fetse, J.P., Kofie, W. and Adosraku, R.K. (2016) Ethnopharmacological Importance of Xylopia aethiopica (dunal) a. Rich (Annonaceae)-A Review. British Journal of Pharmaceutical Research, 11, 1-21. https://doi.org/10.9734/BJPR/2016/24746

[6] Muanya, C. (2008) How Plants Can Induce Labour, Heal After-Birth Wounds. The Guardian Newspaper, 17th Jan. 2008.

[7] Ezekwesili, C.N., Nwodo, O.F.C., Eneh, F.U. and Ogbunugafor, H.A. (2010) Investigation of the Chemical Composition and Biological Activity of Xylopia aethiopica Dunal (Annonacae). African Journal of Biotechnology, 9, 7352-7356.

[8] Onyebuagu, P.C., Kiridi, K. and Aloamaka, C.P. (2014) Effect of Dietary Xylopia aethiopica on the Gonads of Male Wistar Rats. International Journal of Herbs and Pharmacological Research, 3, 40-45.

[9] Hassan, A.M., Almagboul, A.Z. and Kabbashi, A.S. (2016) Chemical Compositions and Cytotoxic Activity of Fruits Essential Oil from Xylopia aethiopica (Dunal) A. Rich. International Journal of Multidisciplinary Research and Development, 3, 394-399.

[10] Erhirhie, E.O. and Moke, G.E. (2014) Xylopia aethiopica: A Review of Its Ethnomedicinal, Chemical and Pharmacological Properties. American Journal of Pharmtech Research, 4, 22-37.

[11] Uzodike, E.B. and Onuoha, I.N. (2010) The Effect of Xylopia aethiopica (Uda) on Intraocular Pressure. Journal of the Nigerian Optometric Association, 16, 21-25. https://doi.org/10.4314/jnoa.v16i1.56629

[12] Obodo, B.N., Iweka, F.K., Obhakhan, J.O., Dada, F.L., Festus, O.O. and Onoyovwi, A.O. (2013) Hepatic Potentials of Xylopia aethiopica Leaves in Adult Wistar Rats. International Journal of Herbs and Pharmacological Research, 2, 36-41.

[13] Chris-Ozoko, L.E., Ekundina, V. and Winiki, C. (2015) Histomorphological Effects of Xylopia aethiopica on the Liver and Kidney of Albino Wistar Rats. Scholars Academic Journal of Biosciences, 3, 150-154.

[14] Elhassan, I.A. and Ayoub, S.M.H. (2014) Effects of Geographic Location on Essential Oil Content and Composition of Xylopia aethiopica. American Journal of Research Communication, 2, 251-261.

[15] Noudjou, F., Kouninki, H., Hance, T., Haubruge, E., Ngamo, L.S.T. and Maponmestsem, P.M. (2007) Composition of Xylopia aethiopica (Dunal) A. Rich Essential Oils from Cameroon and Identification of a Minor Diterpene: [Ent-13-epimanoyl oxide]. Biotechnology and Agronomy of Social Environment, 11, 193-199.

[16] Konan, N.D., Bosson, A.K., Mamyrbekova-Bekro, J.A., Jean, N. and Bekro, Y.A. (2009) Chemical Composition and Antioxidant Activities of Essential Oil of Xylopia aethiopica (Dunal) A. Rich. European Journal of Scientific Research, 2, 311-318.

[17] Olonisakin, A.M., Oladimeji, O. and Lajide, L. (2007) Composition and Antibacterial Activity of Steam Distilled Oils of Xylopia aethiopica and Syzigium aromaticum. Journal of Engineering and Applied Sciences, 2, 236-240. 
[18] Koba, K., Sanda, K., Raynaud, C., Guyon, C., Chaumont, J.P. and Nicod, L. (2008) Chemical Composition and in Vitro Cytotoxic Activity of Xylopia aethiopica (Dun) A. Rich. (Annonaceae) Fruit Essential Oil from Togo. Journal of Essential Oil Research, 20, 354-357. https://doi.org/10.1080/10412905.2008.9700029

[19] EL-Kamali, H.H. and Adam, H.O. (2009) Aromatic Plants from the Sudan: Part II. Chemical Composition of the Essential Oil of Xylopia aethiopica (Dunal) A. Rich. Existence of Chemotype Species. Advances in Natural and Applied Sciences, 3, 166-169.

[20] Apenteng, J.A., Ogundeyi, M., Oppong, E.E., Osei-Asare, C. and Brookman-Amissah, M.G. (2016) In Vitro Anti-Infective and Antioxidant Activity of $X Y$ lopia aethiopica [Dun.] A. Rich: A Comparison of the Fruits and Leaves Extracts. Journal of Medicinal Plants Studies, 4, 24-29.

[21] Sujayil, T.K. and Dhanaraj, T.S. (2016) Determination of Bioactive Compounds in Evolvulus alsinoides Leaf Extract using GC-MS Technique. Research Journal of Life Sciences, Bioinformatics, Pharmaceutical and Chemical Sciences, 2, 34-38.

[22] Gnanavel, V. and Saral, A.M. (2013) GC-MS Analysis of Petroleum Ether and Ethanol Leaf Extracts from Abrus precatorius linn. International Journal of Pharma and Bioscience, 4, 37-44.

[23] Santhosh, K.S., Samydurai, P., Ramakrishnan, R. and Nagarajan, N. (2014) Gas Chromatography and Mass Spectrometry Analysis of Bioactive Constituents of Adiantum Capillus Veneris L. International Journal of Pharmacy and Pharmaceutical Sciences, 6, 60-63.

[24] Mamza, U.T., Sodipo, O.A. and Khan, I.Z. (2012) Gas Chromatography-Mass Spectrometry (GC-MS) Analysis of Bioactive Components of Phyllanthus amarus Leaves. International Research Journal of Plant Science, 3, 208-215.

[25] Okereke, S.C., Arunsi, U.O. and Nosiri, C.I. (2017) GC-MS/FT-IR Screening of $X y$ lopia aethiopica (Dunal) A. Rich Fruit. African Journal of Biochemistry Research, 11, 12-17.

[26] Telascrea, M., de Araújo, C.C., Marques, M.O.M., Facanali, R., de Moraes, P.L.R. and Cavalheiro, A.J. (2007) Essential Oil Leaves of Cryptocaryam andioccana Meisner (Lauraceae): Composition and Intraspecific Chemical Variability. Biochemical System and Ecology, 35, 222-232. https://doi.org/10.1016/j.bse.2006.09.015

[27] Kiran, S.R. and Devi, P.S. (2007) Evaluation of Mosquitocidal Activity of Essential Oil and Sesquiterpenes from Leaves of Chloroxylon swietenia DC. Parasitology Research, 101, 413-418. https://doi.org/10.1007/s00436-007-0485-Z

[28] Hamadan, D.I., Abdulla, R.H., Mohamed, M.E. and El-Shazly, A.M. (2013) Chemical Composition and Biological Activity of Essential Oils of Cleopatra mandarin (Citrus reshni) Cultivated in Egypt. Journal of Pharmacognosy and Phytotherapy, 5, 83-89.

[29] Bruce, T.J.A., Birkett, M.A., Blande, J., Hooper, A.M., Martin, J.L., Khambay, B., Prosser, I., Smart, L.E. and Wadhams, L.J. (2005) Response of Economically Important Aphids to Components of Hemizygia petiolata Essential Oil. Pest Management Science, 61, 1115-1121. https://doi.org/10.1002/ps.1102

[30] Birkett, M.A., Al Abassi, S., Krober, T., Chamberlain, K., Hooper, A.M., Guerin, P.M., Pettersson, J., Pickett, J.A., Slade, R. and Wadhams, L.J. (2008) Antiectoparasitic Activity of the Gum Resin, Gum Haggar, from the East Africa Plant, Commiphor aholtziana. Phytochemical, 69, 1710-1715.

https://doi.org/10.1016/j.phytochem.2008.02.017 
[31] Prakasia, P.P. and Nair, A.S. (2015) Chemical Fingerprint of Essential Oil Components from Fresh Leaves of Glycosmis pentaphylla (Retz.). Correan Pharmaceutical Innovation Journal, 3, 50-56.

[32] Soh, D., Nkwengoua, E., Ngantchou, I., Nyasse, B., Denier, C. and Hannaert, V. (2013) Xylopioxyde and Other Bioactive Kaurane-Diterpenes from Xylopia aethiopica Dunal (Annonaceae). Journal of Applied Pharmaceutical Science, 3, 13-19.

[33] Enemchukwu, B.N., Erimujor, S.O. and Ubaoji, K.I. (2014) Phytochemical Screening and Biochemical Effects of Aqueous Seed Extract of Xylopia aethiopica (uda) on Selected Haematological Indices in Male Wistar Albino Rats. The Bioscientist, 2, 103-109.

[34] Abd-Alg, N.N., El-Kamali, H.H., Ramadan, M.M., Ghanem, K.Z. and Farrag, A.R.H. (2013) Xylopia aethiopica Volatile Compounds Protect against Panadol-Induced Hepatic and Renal Toxicity in Male Rats. World Applied Sciences Journal, 27, 10-22.

[35] Chemical Economics Handbook (CEH) (2016) Cyclohexanol and Cyclohexanone. https://www.ihs.com/products/cyclohexanol-chemical-economics-handbook.html

[36] Safety and Risk Management (SRM) (1999) Identification and Listing of Hazardous Waste. http://www.montana.edu/srm/chemical-safety/panduwastecodes.html 